\title{
Intensive care unit admission of obstetric cases: a single centre experience with contemporary update
}

\author{
Vivian KS Ng *, TK Lo, HH Tsang, WL Lau, WC Leung
}

\section{A B S T R A C T}

Objectives: To review the characteristics of a series of obstetric patients admitted to the intensive care unit in a regional hospital in 2006-2010, to compare them with those of a similar series reported from the same hospital in 1989-1995 and a series reported from another regional hospital in 1998-2007.

Design: Retrospective case series.

Setting: A regional hospital in Hong Kong.

Patients: Obstetric patients admitted to the Intensive Care Unit of Kwong Wah Hospital from 1 January 2006 to 31 December 2010.

Results: From 2006 to 2010, there were 67 such patients admitted to the intensive care unit $(0.23 \%$ of total maternities and $2.34 \%$ of total intensive care unit admission), which was a higher incidence than reported in two other local studies. As in the latter studies, the majority were admitted postpartum $(n=65,97 \%)$, with postpartum haemorrhage $(n=39$, $58 \%$ ) being the commonest cause followed by preeclampsia/eclampsia $(n=17,25 \%)$. In the current study, significantly more patients had had elective caesarean sections for placenta praevia but fewer had had a hysterectomy. The duration of intensive care unit stay was shorter (mean, 1.8 days) with fewer invasive procedures performed than in the two previous studies, but maternal and neonatal mortality was similar (3\% and 6\%, respectively).

Conclusion: Postpartum haemorrhage and pregnancy-induced hypertension were still the most common reasons for intensive care unit admission. There was an increasing trend of intensive care unit admissions following elective caesarean section for placenta praevia and for early aggressive intervention of pre-eclampsia. Maternal mortality remained low but had not decreased. The intensive care unit admission rate by itself might not be a helpful indicator of obstetric performance.

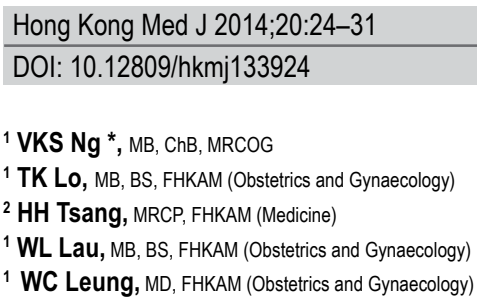

*Corresponding author: vivian_nks@hotmail.com

\section{New knowledge added by this study \\ - There was an increasing trend of obstetric intensive care unit (ICU) admissions but with shorter stays. \\ - Well-planned fertility-sparing treatments for postpartum haemorrhage and placenta praevia may decrease resorting to hysterectomy. \\ Implications for clinical practice or policy \\ - Other performance indicators in addition to crude ICU admission rates should be established to evaluate obstetric standards.}

\section{Introduction}

Obstetric admissions to the intensive care unit (ICU) and maternal mortality continue to have a significant impact on maternal health care, despite the low rate of such admissions in developed countries. ${ }^{1}$ Unlike others, obstetric patients pose a major management challenge to ICU physicians and obstetricians due to altered physiology during pregnancy, consideration of fetal wellbeing, and the unique type of disorders to be dealt with.

Despite ongoing improvements in obstetric care, more patients were admitted to ICU in the reviewed period compared with decades earlier. ${ }^{2}$
Thus, the purpose of this study was to review and compare the characteristics of obstetric patients admitted to the ICU over the recent 20 years using historical controls, with respect to their epidemiology, medical background, antenatal and peripartum risks, durations of ICU stay, interventions in the ICU, and predictability of the Acute Physiology and Chronic Health Evaluation (APACHE II) score, as well as maternal and fetal outcomes.

\section{Methods}

This was a retrospective case series of obstetric patients admitted to the ICU of Kwong Wah 
Hospital, Hong Kong, over a 5-year period from 1 January 2006 to 31 December 2010. Our hospital provides joint care with seven other hospitals in the Kowloon West Cluster to residents of six districts, which account for about 1.9 million inhabitants. Our obstetric service is available for 24 hours each day for women in parts of the Kowloon West and Wong Tai Sin districts. We provide out-patient and in-patient services, including antenatal check-ups, prenatal diagnoses, elective and emergency operations and services that are supported by a blood bank and various laboratory test facilities available for patients in hospital and in the community. Moreover, 24-hour midwifery, and perinatal and anaesthetic services are available in our delivery suite. Our team consists of consultants, associate consultants, as well as senior and junior residents. Three staff (one specialist, two residents) are always available on site for emergency admissions. Annually, we manage 5000 to 6000 deliveries, which is one of the highest delivery rates for a Hong Kong hospital. Our ICU was established in 1968, currently has 14 beds, and admits 500 to 600 patients every year. The ICU team consists of a critical care physician, a resident anaesthetist, medical and surgical residents, and a nursing team with critical care-registered nurse specialists.

This study was approved by the Ethics Committee of the Kowloon West Cluster, Hospital Authority. No patient consent was required as the study only involved review of medical records.

Obstetric patients from 24 weeks of gestation onwards to 6 weeks postpartum admitted to the ICU were reviewed. They were identified via the computerised database system adopted by the ICU. All corresponding medical records were reviewed in detail. Supplementary information was retrieved from the Clinical Management System, Electronic Patient Record, and Obstetrics Clinical Information System.

Data retrieved for analysis included patient demographics (age, ethnicity, smoking and drinking status, parity, order of pregnancy, and body mass index [BMI] at booking visit), antenatal booking status, number of antenatal visits, medical history, perinatal risks, gestation at and mode of delivery, indications for caesarean section, interventions involved at and after delivery, indications and admission status to the ICU, and maternal and fetal outcomes. Patient mortality was predicted by recourse to the APACHE II score.

Indications for ICU admission were divided into obstetric and non-obstetric causes. Obstetric causes were those unique to pregnancy or liable to occur within 6 weeks of delivery. Non-obstetric causes were those not specifically related in pregnancy.

Interventions provided by ICU physicians were classified into non-invasive and invasive. Those deemed non-invasive included insertion of arterial or central lines, blood product transfusion,

\section{入住深切治療部的產科病例：一個中心的 經驗與更新 \\ 吳坤蒨、盧子健、曾憲雄、劉偉霖、梁永昌}

目的：回顧2006至2010年入住一所分區醫院深切治療部的產科病 例, 並把她們與分別於1989至1995年在同一家醫院以及另一所分區 醫院於 1998 至 2007 年的同類病人作比較。

設計：回顧性病例系列。

安排：香港一所分區醫院。

患者：2006年1月 1日至2010年12月31日期間入住廣華醫院深切治療 部的產科病人。

結果：2006至2010年期間共67名產科病人入住深切治療部（佔產科 總額的 $0.23 \%$ 和深切治療部入住率的 $2.34 \%$ ), 患病率高於其餘兩個同 類型本地研究。在兩個同類型的本地研究中, 大多數病人均為產後入 住（ $\mathrm{n}=65 ， 97 \%) ，$ 其中產後出血是最常見的原因（ $\mathrm{n}=39 ， 58 \%)$

其次是子癇前症或子癇症 $(n=17,25 \%)$ 。本研究中, 病人因胎盤前 置而選擇剖腹產的明顯較多, 而子宮切除術則較少。與兩個同類型的 本地研究比較, 本研究的病人入住深切治療部的時間較短 (平均為 1.8 日 ), 且較少進行侵入性程序, 但產婦和新生兒的死亡率卻相近（分 別為3\%和6\%）。

結論 : 產後出血和妊娠高血壓仍然是最常見入住深切治療部的原因。 因胎盤前置而選擇剖腹產以及因子痌前症而進行早期積極干預後, 最 後導致入住深切治療部的有上升的趨勢。產婦死亡率仍然偏低, 但沒 有下降跡象。深切治療部的入住率本身可能不是一個有用的產科表現 指標。

use of continuous positive airway pressure (CPAP) ventilation, and use of inotropes. Invasive procedures included invasive mechanical ventilation, cardiopulmonary resuscitation (CPR), defibrillation, and haemodialysis.

Immediate and long-term complications of the mothers and neonates were assessed up to 6 to 8 weeks post-delivery. Maternal and perinatal mortalities were also calculated.

Data were entered manually into Excel and analysed using the Statistical Package for the Social Sciences (SPSS version 17, Chicago [IL], US). The data were compared with those from the results of a historical review in the same hospital (1989-1995, by Tang et $\mathrm{al}^{2}$ ) and a review in another regional hospital (1998-2007, by Leung et $\mathrm{al}^{3}$ ). Chi squared or Fisher's exact tests were used to compare proportions and Student's $t$ test to compare continuous variables.

\section{Results}

In all, 67 relevant patients were admitted to the ICU and reviewed during the period of 1 January 2006 to 31 December 2010, which amounted to $0.23 \%$ of the total hospital maternities and $2.34 \%$ of all ICU admissions (Table 1). Their demographic features are shown in Table 2 . The mean age of women at delivery was 34 (standard deviation [SD], 5; range, 
20-42) years. Thirty (45\%) of the patients were of advanced maternal age (ie age at confinement of $\geq 35$ years). The majority of them were Chinese ( $n=65$, 97\%), one was Filipino and one an Indonesian. Nine (13\%) patients were visitors from mainland China. Seven (10\%) were smokers, two (3\%) drank alcohol regularly and two (3\%) had a history of substance abuse. In all, 38 (57\%) were nulliparous and six (9\%) carried twin pregnancies. Most of the patients $(\mathrm{n}=61,91 \%)$ were booked in our unit; 19 (28\%) had three antenatal check-ups or less.

Among these 67 patients, 39 (58\%) enjoyed good past health, and six (9\%) had a BMI of more than $25 \mathrm{~kg} / \mathrm{m}^{2}$ at their booking visit. The most common co-existing diseases were gynaecological $(\mathrm{n}=11,16 \%)$ and haematological $(n=9,13 \%)$. Their antenatal and peripartum risks are summarised in Table 3.

The mean gestational age at delivery was 37 (SD, 6; range, 27-41) weeks. Most of them were delivered by emergency caesarean section $(n=34,51 \%)$, including one transferred to us after delivery in the private sector. One patient remained undelivered and died antenatally. Placenta praevia and pregnancy-induced hypertensive disorders were the main indications for elective and emergency caesarean sections, respectively. Other indications are listed in Table 4.

The reasons for ICU admission and procedures undertaken therein are listed in Table 5. Most were admitted to the ICU postpartum $(n=65,97 \%)$ and for obstetric problems ( $n=58,87 \%)$, of which postpartum haemorrhage $(\mathrm{PPH})$ was the leading cause $(\mathrm{n}=39$, $58 \%$ ) followed by pre-eclamptic toxaemia (PET) or eclampsia $(n=17,25 \%)$. The mean duration of ICU stay was 1.8 (SD, 1.2; range, $0.5-10)$ days; four (6\%) of the patients stayed for more than 3 days.

In all, 39 patients were admitted to the ICU due to a PPH, the mean estimated blood loss was $4852 \mathrm{~mL}$. Major causes of PPH were related to placenta praevia $(n=16)$, uterine atony $(n=12)$, and perineal trauma $(n=5)$. Blood products given included packed cells (mean, 12 units), platelet concentrate (mean, 5 units), fresh frozen plasma (mean, 7 units), and cryoprecipitate (mean, 1 unit). Three patients received recombinant factor VIIa (NovoSeven; Novo Nordisk A/S, Bagsværd, Denmark). Procedures to control PPH included compression sutures $(n=10)$, uterine artery embolisation $(n=9)$, insertion of a

TABLE I. Intensive care unit (ICU) admission of obstetric patients

\begin{tabular}{|c|c|c|c|c|c|}
\hline & $\begin{array}{l}\text { Tang et al's } \\
\text { study }^{*}\end{array}$ & $\begin{array}{l}\text { Leung et al's } \\
\text { study }^{*}\end{array}$ & Our study* & $\begin{array}{l}\text { P value } \\
\text { (our study } \\
\text { vs Tang et } \\
\text { al's study') }\end{array}$ & $\begin{array}{c}\text { P value } \\
\text { (our study } \\
\text { vs Leung et } \\
\text { al's study') }\end{array}$ \\
\hline ICU admission rate (No./total maternities) & $0.12 \%(49 / 39354)$ & $0.13 \%(50 / 37505)$ & $0.23 \%(67 / 28976)$ & 0.001 & 0.003 \\
\hline ICU utilisation rate (No./total ICU admission) & $0.61 \%(49 / 8032)$ & $0.65 \%(50 \dagger / 7692)$ & $2.34 \%(67 / 2868)$ & $<0.001$ & $<0.001$ \\
\hline Duration of admission (days) & $4.1 \pm 2.3(1-19)$ & 2 & $1.8 \pm 1.2(0.5-10)$ & $<0.001$ & - \\
\hline Stay $>3$ days & $23(47 \%)$ & $\mathrm{N} / \mathrm{A}$ & $4(6 \%)$ & $<0.001$ & - \\
\hline \multicolumn{6}{|l|}{ Admission status } \\
\hline Antepartum & $6(12 \%)$ & $11(22 \%)$ & $2(3 \%)$ & 0.052 & 0.001 \\
\hline Postpartum & $43(88 \%)$ & $39(78 \%)$ & $65(97 \%)$ & - & - \\
\hline Elective admission (booked before admission to ICU) & $\mathrm{N} / \mathrm{A}$ & $6(12 \%)$ & $10(15 \%)$ & N/A & 0.649 \\
\hline Emergency admission & N/A & $44(88 \%)$ & 57 (85\%) & - & - \\
\hline
\end{tabular}

Abbreviation: N/A = data not available

* Data are shown as \%, No. (\%), or mean \pm standard deviation (range)

$\dagger 6$ Patients were less than 24 weeks of gestation

TABLE 2. Basic demographics

\begin{tabular}{|c|c|c|c|c|c|}
\hline Demographics & $\begin{array}{l}\text { Tang et al's } \\
\text { study }^{*}\end{array}$ & $\begin{array}{l}\text { Leung et al's } \\
\text { study }^{3 *}\end{array}$ & Our study* & $\begin{array}{l}\text { P value (our study vs } \\
\text { Tang et al's study') }\end{array}$ & $\begin{array}{l}\text { P value (our study vs } \\
\text { Leung et al's study) }\end{array}$ \\
\hline Maternal age at delivery (years) & $32 \pm 5(21-42)$ & $31 \pm 6$ & $34 \pm 5(20-42)$ & 0.012 & 0.004 \\
\hline Advanced maternal age ( $\geq 35$ years) & $14(29 \%)$ & $9(18 \%)$ & $30(45 \%)$ & 0.076 & 0.002 \\
\hline Non-booked & $15(31 \%)$ & $14(28 \%)$ & $6(9 \%)$ & 0.003 & 0.007 \\
\hline Primigravida & $20(41 \%)$ & $\mathrm{N} / \mathrm{A}$ & $38(57 \%)$ & 0.09 & $\mathrm{~N} / \mathrm{A}$ \\
\hline Twin pregnancy & N/A & N/A & 6 & $\mathrm{~N} / \mathrm{A}$ & $\mathrm{N} / \mathrm{A}$ \\
\hline Obesity (BMI $\geq 25$ kg/m²) & $\mathrm{N} / \mathrm{A}$ & $\mathrm{N} / \mathrm{A}$ & $6(9 \%)$ & $\mathrm{N} / \mathrm{A}$ & $\mathrm{N} / \mathrm{A}$ \\
\hline Gestation at delivery (weeks) & $36 \pm 4$ & $34 \pm 9(6-44)$ & $37 \pm 6(27-41)$ & 0.987 & 0.049 \\
\hline Previous caesarean section & $7(14 \%)$ & N/A & $15(22 \%)$ & 0.272 & $\mathrm{~N} / \mathrm{A}$ \\
\hline
\end{tabular}

Abbreviations: $\mathrm{BMI}=$ body mass index; N/A = data not available

* Data are shown as No (\%) or mean \pm standard deviation (range) 
TABLE 3. Past health, antenatal and peripartum risks (more than one entry possible)

\begin{tabular}{|c|c|c|c|c|c|}
\hline \multirow[t]{2}{*}{ Health risk } & \multicolumn{3}{|c|}{ No. (\%) } & \multirow{2}{*}{$\begin{array}{l}\text { P value } \\
\text { (our study } \\
\text { vs Tang et } \\
\text { al's study²) }\end{array}$} & \multirow{2}{*}{$\begin{array}{l}\text { P value (our } \\
\text { study vs } \\
\text { Leung et } \\
\text { al's study') }\end{array}$} \\
\hline & Tang et al's study² & Leung et al's study ${ }^{3}$ & Our study & & \\
\hline Good past health & $40(82 \%)$ & $43(86 \%)$ & 39 (58\%) & 0.008 & 0.001 \\
\hline Cardiovascular & $\begin{array}{l}6(12 \%) \text { : Chronic } \\
\text { rheumatic heart } \\
\text { disease }(n=4), \text { chronic } \\
\text { hypertension }(n=2)\end{array}$ & $\begin{array}{l}2(4 \%) \text { : Chronic rheumatic } \\
\text { heart disease }(n=1) \\
\text { hypertension }(n=1)\end{array}$ & $\begin{array}{l}2(3 \%) \text { : Supraventricular } \\
\text { tachycardia }(n=1) \\
\text { non-obstructive } \\
\text { cardiomyopathy }(n=1)\end{array}$ & 0.052 & 0.765 \\
\hline Neurological & $\begin{array}{l}2(4 \%) \text { : Poliomyelitis }(n=1) \\
\text { Segawa disease }(n=1)\end{array}$ & 0 & $\begin{array}{l}4(6 \%) \text { : Epilepsy }(n=3) \\
\text { myasthenia gravis }(n=1)\end{array}$ & 0.65 & - \\
\hline Haematological & 1 (2\%): Aplastic anaemia & 0 & $\begin{array}{l}9(13 \%) \text { : Thalassemia or } \\
\text { anaemia }(n=9)\end{array}$ & 0.031 & - \\
\hline Infection-related & 0 & $\begin{array}{l}5(10 \%) \text { : Hepatitis } B(n=4), \\
\text { pulmonary tuberculosis }(n=1)\end{array}$ & $\begin{array}{l}3(4 \%) \text { : Syphilis }(n=2) \text {, neck } \\
\text { tuberculosis }(n=1)\end{array}$ & - & 0.242 \\
\hline Gynaecological & 0 & $1(2 \%)$ : Uterine fibroid & $\begin{array}{l}11(16 \%): \text { Fibroids or } \\
\text { ovarian cysts }(n=11)\end{array}$ & - & 0.011 \\
\hline Endocrine & 0 & 0 & $\begin{array}{l}4(6 \%) \text { : Diabetes mellitus } \\
(n=2), \text { thyroid disease }(n=2)\end{array}$ & - & - \\
\hline Other & 0 & 0 & $\begin{array}{l}6(9 \%) \text { : Psychiatric disease } \\
(n=4), \text { asthma }(n=1), \text { renal } \\
\text { disease }(n=1)\end{array}$ & - & - \\
\hline \multicolumn{6}{|c|}{ Antenatal and peripartum risk } \\
\hline Placenta praevia & $2(4 \%)$ & N/A & $20(30 \%)$ & $<0.001$ & N/A \\
\hline APH/abruptio placentae & $5(10 \%)$ & N/A & $10(15 \%)$ & 0.454 & N/A \\
\hline $\mathrm{PIH} / \mathrm{PET}$ & $8(16 \%)$ & N/A & $15(22 \%)$ & 0.419 & N/A \\
\hline GDM & $9(18 \%)$ & N/A & $22(33 \%)$ & 0.082 & N/A \\
\hline
\end{tabular}

Abbreviations: $\mathrm{APH}=$ antepartum haemorrhage; $\mathrm{PIH}$ = pregnancy-induced hypertension; $\mathrm{PET}$ = pre-eclampsia toxaemia; $\mathrm{GDM}=$ gestational diabetes; $\mathrm{N} / \mathrm{A}=$ data not available

TABLE 4. Mode of delivery

\begin{tabular}{|c|c|c|c|c|c|}
\hline & $\begin{array}{l}\text { Tang et al's } \\
\text { study }^{2}\end{array}$ & $\begin{array}{l}\text { Leung et al's } \\
\text { study }^{3}\end{array}$ & Our study & $\begin{array}{l}\text { P value (our study vs } \\
\text { Tang et al's study') }\end{array}$ & $\begin{array}{l}\text { P value (our study vs } \\
\text { Leung et al's study }{ }^{3} \text { ) }\end{array}$ \\
\hline NSD & $13(27 \%)$ & $9(18 \%)$ & $9(13 \%)$ & 0.076 & 0.498 \\
\hline Instrumental delivery & $2(4 \%)$ & $3(6 \%)$ & $6(9 \%)$ & 0.306 & 0.553 \\
\hline Elective caesarean section & $3(6 \%)$ & $6(12 \%)$ & $17(25 \%)$ & 0.007 & 0.072 \\
\hline Emergency + crash caesarean section & $30(61 \%)$ & $32(64 \%)$ & $34(51 \%)$ & 0.262 & 0.153 \\
\hline Undelivered & $1(2 \%)$ & 0 & $1(2 \%)$ & 0.823 & - \\
\hline \multicolumn{6}{|l|}{ Indication for caesarean section } \\
\hline \multicolumn{6}{|l|}{ Elective caesarean section } \\
\hline Placenta praevia & 3 & $\mathrm{~N} / \mathrm{A}$ & 13 & 0.04 & $\mathrm{~N} / \mathrm{A}$ \\
\hline \multicolumn{6}{|c|}{ Emergency + crash caesarean section (multiple entries) } \\
\hline PIH (including PET/ eclampsia) & 4 & $\mathrm{~N} / \mathrm{A}$ & 12 & 0.176 & $\mathrm{~N} / \mathrm{A}$ \\
\hline Placenta praevia & 0 & $\mathrm{~N} / \mathrm{A}$ & 3 & - & $\mathrm{N} / \mathrm{A}$ \\
\hline $\mathrm{APH}$ & 5 & $\mathrm{~N} / \mathrm{A}$ & 5 & 0.603 & $\mathrm{~N} / \mathrm{A}$ \\
\hline Fetal distress & 9 & N/A & 1 & 0.001 & $\mathrm{~N} / \mathrm{A}$ \\
\hline Breech & 1 & $\mathrm{~N} / \mathrm{A}$ & 2 & 0.752 & $\mathrm{~N} / \mathrm{A}$ \\
\hline No progress in labour/CPD & 8 & $\mathrm{~N} / \mathrm{A}$ & 6 & 0.229 & $\mathrm{~N} / \mathrm{A}$ \\
\hline Uterine scar related & 1 & $\mathrm{~N} / \mathrm{A}$ & 3 & 0.477 & $\mathrm{~N} / \mathrm{A}$ \\
\hline Surgical/medical diseases & 2 & $\mathrm{~N} / \mathrm{A}$ & 2 & 0.749 & $\mathrm{~N} / \mathrm{A}$ \\
\hline Obstetric emergency & 1 (Scar rupture) & $\mathrm{N} / \mathrm{A}$ & 1 (Cord prolapse) & 0.823 & $\mathrm{~N} / \mathrm{A}$ \\
\hline
\end{tabular}

Abbreviations: NSD = normal spontaneous delivery; $\mathrm{PIH}$ = pregnancy-induced hypertension; $\mathrm{PET}$ = pre-eclampsia toxaemia; $\mathrm{APH}=$ antepartum haemorrhage; $\mathrm{CPD}=$ cephalopelvic disproportion; N/A = data not available 
Sengstaken-Blakemore tube $(n=6)$, and uterine artery ligation $(n=2)$. There were 13 patients who underwent hysterectomy despite multiple other interventions and use of multiple uterotonics, and eight patients with $\mathrm{PPH}$ were complicated with disseminated intravascular coagulation, one had a ventricular tachycardia, and one had a urinary tract injury. One of the patients with a PPH and anaphylactic shock suffered a cavernous sinus thrombosis and a cranial nerve VI palsy, for which she received therapeutic doses of low-molecular-weight heparin. Another patient was admitted 2 weeks after delivery due to delirium secondary to sepsis.

There were 17 patients admitted to the ICU for PET or eclampsia (9 of whom had eclampsia) and were all stabilised in the ICU. Two patients had HELLP (haemolysis, elevated liver enzymes, low platelets) syndrome and two had hypertensive encephalopathy diagnosed on the basis of computed tomography. Other complications included acute pulmonary oedema $(n=1)$, deranged renal function $(n=3)$, deranged liver function $(n=1)$, and aspiration pneumonitis $(n=1)$. Another eight had persistent hypertension 6 weeks postpartum and were referred to physicians.

Regarding the 10 patients (15\% of the cohort) admitted to the ICU for non-obstetric reasons, two had an epileptic seizure, three had cardiovascular problems (cardiomyopathy, heart failure, and pulmonary hypertension), and one each had renal disease, ethanol toxicity, acute pulmonary oedema, myasthenia gravis, and anaphylactic shock.

Invasive procedures performed in the ICU were CPR $(n=2,3 \%)$ and mechanical ventilation $(n=7$, $10 \%)$. Non-invasive procedures were blood product transfusions $(\mathrm{n}=36,54 \%)$, central line insertion $(n=18,27 \%)$, arterial line insertion $(n=24,36 \%)$, use of inotropes $(n=2,3 \%)$, and CPAP ventilation $(n=1,2 \%)$.

The mean APACHE II score was 17 (range, 4-37) and the mean predicted mortality rate was $28 \%$ (range, $4-85 \%$ ). The actual mortality rate in this series was $3 \%$ (Table 6). The maternal mortality ratio (MMR; actual/predicted mortality) was 0.11 .

TABLE 5. Reasons for intensive care unit (ICU) admission

\begin{tabular}{|c|c|c|c|c|c|}
\hline \multirow[t]{2}{*}{ Cause } & \multicolumn{3}{|c|}{ No $(\%)$ or mean \pm standard deviation (range) } & \multirow{2}{*}{$\begin{array}{l}\text { P value (our } \\
\text { study vs Tang } \\
\text { et al's study²) }\end{array}$} & \multirow{2}{*}{$\begin{array}{l}\text { P value (our } \\
\text { study vs Leung } \\
\text { et al's study }{ }^{3} \text { ) }\end{array}$} \\
\hline & $\begin{array}{c}\text { Tang et } \\
\text { al's study² }\end{array}$ & $\begin{array}{l}\text { Leung et } \\
\text { al's study }\end{array}$ & Our study & & \\
\hline Obstetric & 33 & 35 & 58 & 0.041 & 0.068 \\
\hline Non-obstetric & 16 & 15 & 10 & - & - \\
\hline \multicolumn{6}{|l|}{ Obstetric causes ${ }^{*}$} \\
\hline $\mathrm{PPH}$ & $26(53 \%)$ & $19(38 \%)$ & $39(58 \%)$ & 0.581 & 0.031 \\
\hline Mean blood loss (mL) & $3500 \pm 1400(1000-8500)$ & 5200 & $4852 \pm 4903(300-25500)$ & 0.064 & - \\
\hline PPH with hysterectomy & 22 (85\%) & 7 (37\%) & $13(33 \%)$ & $<0.001$ & 0.792 \\
\hline PET/eclampsia & $7(14 \%)$ & 7 (14\%) & $17(25 \%)$ & 0.145 & 0.132 \\
\hline Others & 0 & $9(18 \%) \dagger$ & $3(5 \%) \ddagger$ & - & 0.017 \\
\hline \multicolumn{6}{|l|}{ Non-obstetric causes* } \\
\hline Medical & $7(14 \%)$ & $14(28 \%)$ & $8(12 \%)$ & 0.895 & 0.028 \\
\hline Anaesthesia & $7(14 \%)$ & 0 & $1(2 \%)$ & 0.007 & - \\
\hline Surgical & $2(4 \%)$ & $1(2 \%)$ & 0 & - & - \\
\hline Psychiatric & 0 & 0 & $1(2 \%)$ & - & - \\
\hline \multicolumn{6}{|l|}{ Procedures in ICU } \\
\hline \multicolumn{6}{|l|}{ Invasive } \\
\hline Intubation & $21(43 \%)$ & $29(58 \%)$ & $7(10 \%)$ & $<0.001$ & $<0.001$ \\
\hline CPR & 0 & 0 & $2(3 \%)$ & - & - \\
\hline \multicolumn{6}{|l|}{ Non-invasive } \\
\hline Blood product transfusion & N/A & - & $36(54 \%)$ & - & - \\
\hline Central venous line insertion & N/A & $26(52 \%)$ & $18(27 \%)$ & - & 0.005 \\
\hline Arterial line insertion & N/A & $33(66 \%)$ & $24(36 \%)$ & - & 0.001 \\
\hline Inotropes & N/A & $8(16 \%)$ & $2(3 \%)$ & - & 0.013 \\
\hline Non-invasive mechanical ventilation & N/A & $3(6 \%)$ & $1(2 \%)$ & - & 0.184 \\
\hline
\end{tabular}

Abbreviations: $\mathrm{PPH}=$ postpartum haemorrhage; $\mathrm{PET}$, = pre-eclampsia toxaemia; $\mathrm{CPR}=$ cardiopulmonary resuscitation; $\mathrm{N} / \mathrm{A}=$ data not available

* One patient in our study had both obstetric and non-obstetric indications; one patient had 2 obstetric indications

+ Amniotic fluid embolism $(n=3)$, ruptured ectopic pregnancy $(n=2)$, peripartum cardiomyopathy $(n=1)$, antepartum haemorrhage $(n=1)$, septic abortion $(n=1)$, and retained placenta with shock $(n=1)$

‡ Amniotic fluid embolism $(n=1)$, intrapartum sepsis $(n=1)$, and placenta praevia with retained placenta $(n=1)$ 
In our study period, there were two maternal deaths in the 28976 maternities or 7 per 100000 births, both in ICU patients. One was a patient who enjoyed good health but suspected to have pulmonary hypertension at 27 weeks of gestation, who rapidly deteriorated and died 1 day after admission. Her diagnosis was confirmed at postmortem examination. The other maternal death ensued in the postpartum period due to multi-organ failure and brain death, secondary to eclampsia and intraventricular haemorrhage.

Regarding these ICU admissions, three (5\%) of the fetuses endured intrauterine death (IUD) and one $(2 \%)$ whose neonate died (due to necrotising enterocolitis). The IUDs were associated with abruptio placentae, pulmonary hypertension, and severe preeclampsia with early intrauterine growth restriction.

\section{Discussion}

The health care system of Hong Kong aims to protect/ improve maternal and child health, by means of antenatal, intrapartum, and postnatal services that are readily available at very low costs. Whilst the MMR fluctuated between 1.0 and 11.2 per 100000 live births over the past 31 years, ${ }^{4,5}$ the above-mentioned services have contributed to the decreasing and now very low maternal mortality rates.

Despite advances in obstetric care, the admission rate to the ICU had doubled compared with a decade ago (from $0.12 \%$ to $0.23 \%$ ). ${ }^{2}$ Whereas such ICU utilisation rates for obstetric cases were also higher compared with Tang et al's data ${ }^{2}(2.34 \%$ vs $0.61 \%)$, nevertheless they were low compared to reports from overseas. ${ }^{6,7}$ The rates were also higher than those reported by Leung et al (admission, $0.13 \%$; utilisation, $0.65 \%){ }^{3}$ One of the reasons for the rise in ICU admission rates was changes in patient allocation in our hospital, and over the Hong Kong Special Administrative Region. The number of beds in our ICU was reduced from 18 to 14 after the severe acute respiratory syndrome epidemic in 2003. The number of surgical admissions was also much lower than a decade earlier. Moreover, the number of trauma cases dropped significantly, since two other nearby tertiary hospitals became trauma centres.
Changing attitudes of obstetricians and anaesthetists also contributed to the increase in ICU admission rate. Given the fact that our patients were most commonly delivered by elective caesarean section for placenta praevia, a proper preoperative management plan with a multidisciplinary approach involving anaesthetist, intensive care physician, and obstetricians should have been available before the operation, which included booking of the ICU bed. With the increasing trend of placenta praevia, it was expected that more and more patients would be admitted to the ICU electively for monitoring rather than any future active intervention. The shorter duration of ICU stays, compared with those detailed earlier by Tang et al, ${ }^{2}$ is probably consistent with this trend towards elective admissions.

The mean age of our patients at delivery was higher than that in the patient series described by Tang et $\mathrm{al}^{2}$ and Leung et $\mathrm{al}^{3}{ }^{3}$ Indeed, patients of advanced maternal age were more likely to be admitted to the ICU when compared with our background population, though this was not shown for such ICU admissions reported by Selo-Ojeme et al. $^{8}$ Increasing maternal age implies that our patients were more likely to have co-existing diseases complicating pregnancy, as reflected by our data, even though the medical problems in question were generally mild and stable.

According to Tang et al's ${ }^{2}$ and Leung et al's ${ }^{3}$ reports about non-booked cases (NBCs), patients from mainland China used to be admitted via the emergency department very late when they went into advanced labour. As a result, potential or present obstetric complications were known to us only when they were admitted. With the commencement of the policy to allow these mothers to register and deliver in Hong Kong (since 2007), the number of NBCs decreased significantly, as did their number of ICU admissions.

In the literature there are conflicting data when parity is considered one of the risk factors for ICU admission. In our study, nulliparity was not related to ICU admission, which was also what Pollock et al noted. ${ }^{6}$

During our data analysis, twin pregnancy was more likely in our ICU patients compared with

TABLE 6. Morbidity and mortality

\begin{tabular}{lccccc}
\hline & \multicolumn{2}{c}{ No (\%) or mean \pm standard deviation (range) } & $\begin{array}{c}\text { P value (our study vs } \\
\text { Tang et al's study })\end{array}$ & $\begin{array}{c}\text { P value (our study vs } \\
\text { Leung et al's study }\end{array}$ \\
\cline { 2 - 5 } & Tang et al's study & Leung et al's study & Our study & & - \\
\hline Mean APACHE II score & $13(1-27)$ & $\begin{array}{c}16 \pm 9 \text { (Obstetric) } \\
12 \pm 9 \text { (Non-obstetric) }\end{array}$ & $17 \pm 8(4-37)$ & - & - \\
Mean predicted mortality rate & $18 \%$ & N/A & $28 \%$ & - & - \\
Maternal deaths & $2(4 \%)$ & $3(6 \%)$ & $2(3 \%)$ & 0.749 & - \\
Maternal mortality & 5 per 100000 & 8 per 100000 & 7 per 100000 & - & - \\
Perinatal mortality & $5(10 \%)$ & $4(8 \%)$ & $4(6 \%)$ & 0.400 & 0.667 \\
\hline
\end{tabular}

Abbreviations: $\mathrm{APACHE}=$ Acute Physiology and Chronic Health Evaluation; N/A = data not available 
the background population. However, such data cannot be retrieved from Tang et al's or Leung et al's reports. ${ }^{2,3}$ Twin pregnancy is known to confer a higher risk of gestational diabetes, hypertension, premature delivery, operative deliveries, and postpartum complications (including PPH).,10 Our findings also supported the need of a specialised twin pregnancy clinic to look after this high-risk group.

Placenta praevia was the most frequent risk factor identified in our patient series, being much more common than in Tang et al's study. ${ }^{2}$ Increasing popularity of evaluation by ultrasound has raised the detection rate of placenta praevia early in the antenatal period. All our patients with placenta praevia were delivered electively with proper preoperative arrangements. These entailed booking of ICU facilities, standby uterine artery embolisation, preparation of recombinant factor VIIa and Sengstaken-Blakemore tubes, and involvement of obstetric consultants to make decisions. One consequence was a significantly higher number of elective caesarean sections for placenta praevia compared with decades ago, though the overall section rate remained relatively stable. ${ }^{11}$ This also correlated with placenta praevia being the commonest causes of PPH in our ICU patients.

As in Tang et al's ${ }^{2}$ and Leung and et al's ${ }^{3}$ studies, in our series admissions due to obstetric problems remained the main cause of obstetric ICU admissions. Postpartum haemorrhage was consistently the most common indication for ICU admission, which was also noted in Tang et al's series. ${ }^{2}$ Although the mean estimated blood loss of our patients was apparently higher than that reported by Tang et al, ${ }^{2}$ and abdominal delivery is known to increase the risk of hysterectomy following $\mathrm{PPH},{ }^{12}$ the number of hysterectomies performed was significantly lower than before. The increasing use of compression sutures and uterine artery embolisation together with strategies to retain the placenta in cases of placenta accreta might account for the decreasing recourse to hysterectomy compared with 20 years ago.

The current series had more patients with preeclampsia or eclampsia admitted to the ICU than those reported by Tang et al, ${ }^{2}$ although the difference was not statistically significant. As suggested by the National Institute for Health and Clinical Excellence guideline,${ }^{12}$ our management protocol was updated to incorporate the more liberal use of antihypertensives and magnesium sulphate. Our use of the modified early obstetric warning scoring system allowed early detection of potential complications to prevent poor obstetric outcomes. Intensive care is indicated in patients with severe hypertension, or moderate hypertension with symptoms of impending eclampsia or any suggestion of organ dysfunction. These innovations lead to the rising trend of ICU admissions to monitor for pre-eclampsia.

Active involvement of anaesthetists plays a role in the changing pattern of obstetric ICU admissions. There was a drastic reduction of admissions for anaesthesia-related causes compared with those reported by Tang et al. ${ }^{2}$ Only one of our patients was admitted due to anaphylactic shock, which can be explained by the significant improvements in anaesthetic care and mechanical ventilation in our hospital. Invasive and non-invasive procedures (eg intubation and insertion of arterial and central venous lines) undertaken in the ICU were significantly fewer than decades ago, as most of them had been performed before admission to ICU by anaesthetists. In our series, the mean duration of ICU stay was 1.8 days, which was shorter than 4.1 days reported in Tang et al's study. ${ }^{2}$ The change in attitude and approach to management of both obstetricians and anaesthetists made ICU admission a more elective occurrence than before. As a result, patients admitted to the ICU tended to be more stable and fewer invasive interventions were warranted. These observations highlight the need for obstetric highdependency units to cater for patients requiring more intensive care, but not to the extent of ICU support. ${ }^{13}$

When compared with the findings reported by Leung et $\mathrm{al}^{3}{ }^{3}$ over the decades there was no significant increase in perinatal mortality, nor was there an increased rate of fetal loss when compared with our background population. Nevertheless, maternal mortality had not decreased. In our series, there were two maternal deaths that amounted to a mortality rate of 7 per 100000 maternities. In Tang et al's series ${ }^{2}$ the maternal mortality was 5 per 100000 , and in the UK it was reported to be 14 and 11 per 100000 in 2003-2005 and 2006-2008, respectively. ${ }^{14}$ However, these differences between series were not statistically significant.

One limitation of our study was that data collection from the computerised system might have omitted pregnant women admitted to the ICU from other specialties with diagnoses that were not obstetrically related. A second limitation was that the causes of maternal ICU admission may not relate directly to the causes of maternal mortality. For example, thromboembolism, one of the leading causes of maternal death in the UK, was not a major cause of ICU admission. In the UK, only $30 \%$ of such maternal deaths were in patients admitted to the $\mathrm{ICU},{ }^{15}$ and on this issue there is no global consensus on the optimal indications for ICU admission. A third limitation was that the frequency of obstetric ICU admissions is also affected by the standard of obstetric care and the threshold admission criteria determined by obstetricians, anaesthetists, and intensive care physicians. As a result, ICU admissions may not truly reflect the standard of obstetric care ${ }^{16} \mathrm{~A}$ composite performance indicator of obstetric care by combining the frequency of ICU admission, numbers of emergency admissions and/or proportions of emergency/elective admissions, and proportions 
having prolonged stays (eg $>3$ days) could be a more useful measure of the standard of obstetric practice in the future.

The APACHE II scoring system has been used as a quantitative predictor of mortality, mainly in medical and surgical patients admitted to the ICU using several physiological measures. Its potential has also been evaluated when applied to obstetric patients. According to different overseas reviews, it may overestimate risks in pregnant patients, as in them normal physiology can differ, often in subtle ways, and can undergo abrupt changes in various emergency conditions. ${ }^{17,18}$ Thus, to date, there is still no proper screening system for obstetric emergencies. A specific scoring system for obstetric patients should be developed and warrants a large-scale international prospective study for this purpose.

The majority of patients discharged from our ICU enjoyed satisfactory recoveries in the puerperal period. In all, eight patients with hypertensive disorder in pregnancy had persistent hypertension for which they were referred for medical assessment. In our series, long-term outcome was not determined. Leung et $\mathrm{al}^{3}$ found that women admitted to the ICU had lower mean scores for quality of life than normal Hong Kong females of similar age, but these authors commented that the relationship of low scores to the obstetric illnesses was unclear and might be resolved by long-term patient follow-up.

In some respects, Leung et al's study ${ }^{3}$ also provided us with geographical controls. However, they included patients with gestational ages of $<24$ weeks, which complicated any comparison of risk factors. Moreover, being intensive care physicians they emphasised quality of life after discharge from the ICU. In contrast, we obstetricians looked for indicators to prevent/reduce maternal ICU admissions, as advocated in modern obstetrical care guidelines.

\section{Conclusion}

Our findings illustrate the various changes in ICU admission practice of obstetric cases in the last 20 years, and are comparable to those in other developed countries. Elective caesarean section for placenta praevia and $\mathrm{PPH}$ were the major reasons for ICU admission. More conservative management of placenta praevia and $\mathrm{PPH}$ appeared to reduce resorting to hysterectomy. These ongoing changes in practice may make emergency obstetric admissions to the ICU less likely in the future. Maternal mortality in our unit has remained low over the years, and can hardly be reduced any further. It is therefore more important to refine and improve obstetric practice to reduce maternal morbidity. The 'near-miss' in terms of obstetric ICU admission rates, together with measures targeting the duration of ICU stay, and a potential obstetric morbidity scoring system will no doubt better reflect our clinical performance standards in the future.

\section{References}

1. Public Health Agency of Canada. Make every mother and child count: report on maternal and child health in Canada. 2005. Available from: http://publications.gc.ca/collections/ Collection/H124-13-2005E.pdf. Accessed Jun 2010.

2. Tang LC, Kwok AC, Wong AY, Lee YY, Sun KO, So AP. Critical care in obstetrical patients: an eight-year review. Chin Med J (Engl) 1997;110:936-41.

3. Leung NY, Lau AC, Chan KK, Yan WW. Clinical characteristics and outcomes of obstetric patients admitted to the intensive care unit: a 10-year retrospective review. Hong Kong Med J 2010;16:18-25.

4. Department of Health, Hong Kong. Chapter 1: Health of community. Annual Report 2009/2010.

5. Department of Health, Hong Kong. Number of maternal deaths by major disease group, 2001-2009. Available from: www.healthyhk.gov.hk/phisweb/reports/2009_01_01_ MOT00101.xls. Accessed Aug 2010.

6. Pollock W, Rose L, Dennis CL. Pregnant and postpartum admissions to the intensive care unit: a systematic review. Intensive Care Med 2010;36:1465-74.

7. Togal T, Yucel N, Gedik E, Gulhas N, Toprak HI, Ersoy MO. Obstetric admissions to the intensive care unit in a tertiary referral hospital. J Crit Care 2010;25:628-33.

8. Selo-Ojeme DO, Omosaiye M, Parijat Battacharjee P, Kadir RA. Risk factors for obstetric admissions to the intensive care unit in a tertiary hospital: a case-control study. Arch Gynecol Obstet 2005;272:207-10.

9. Liu AL, Yung WK, Yeung HN, et al. Factors influencing the mode of delivery and associated pregnancy outcomes for twins: a retrospective cohort study in a public hospital. Hong Kong Med J 2012;18:99-107.

10. Yung WK, Liu AL, Lai SF, et al. A specialised twin pregnancy clinic in a public hospital. Hong Kong J Gynaecol Obstet Midwifery 2012;12:21-32.

11. Department of Obstetrics and Gynaecology, Kwong Wah Hospital. Annual Report 2006-2010.

12. National Institute for Health and Clinical Excellence. Caesarean section. NICE Clinical Guidelines, No 132. 2010.

13. Mirghani HM, Hamed M, Ezimokhai M, Weerasinghe DS. Pregnancy-related admissions to the intensive care unit. Int J Obstet Anesth 2004;13:82-5.

14. Cantwell R, Clutton-Brock T, Cooper G, et al. Saving mothers' lives: reviewing maternal deaths to make motherhood safer: 2006-2008. The eighth report on confidential enquiries into maternal deaths in the United Kingdom. BJOG 2011;118(Suppl 1):1S-203S.

15. Lewis G, Clutton-Brock T, Cooper G, et al. Saving mothers' lives: reviewing maternal deaths to make motherhood safer-2003-2005. The seventh report on confidential enquiries into maternal deaths in the United Kingdom. London: CEMACH; 2007.

16. Baskett T, Sternadel J. Maternal intensive care and near-miss mortality in obstetrics. Br J Obstet Gynaecol 1998;105:981-4.

17. Vasquez DN, Estenssoro E, Canales HS, et al. Clinical characteristics and outcomes of obstetric patients requiring ICU admission. Chest 2007;131:718-24.

18. Gilbert TT, Smulian JC, Martin AA, et al. Obstetric admissions to the intensive care unit: outcomes and severity of illness. Obstet Gynecol 2003;102:897-903. 